\title{
The Build-Up of Aerosols Carrying the SARS-CoV-2 Coronavirus, in Poorly Ventilated, Confined Spaces
}

Björn Birnir ( $\square$ birnir@math.ucsb.edu )

University of California, Santa Barbara

\section{Research Article}

Keywords: Respiratory Droplets, Lagrangian Turbulent Air-flow, Virion Decay Time, Polynomial Increase

Posted Date: February 22nd, 2021

DOl: https://doi.org/10.21203/rs.3.rs-110711/v2

License: (c) (i) This work is licensed under a Creative Commons Attribution 4.0 International License. Read Full License 


\title{
The Build-Up of Aerosols Carrying the SARS-CoV-2 Coronavirus, in Poorly Ventilated, Confined Spaces
}

\author{
Björn Birnir \\ Center for Complex and Nonlinear Science \\ Department of Mathematics \\ University of California, Santa Barbara \\ Santa Barbara, CA 93106
}

January 2021

\begin{abstract}
A model of the distribution of respiratory droplets and aerosols by Lagrangian turbulent air-flow is developed and used to show how the SARS-CoV-2 Coronavirus can be dispersed by the breathing of an infected person. It is shown that the concentration of viruses in the exhaled cloud can increase to infectious levels with time (grow linearly), in a confined space where the air re-circulates. The model is used to analyze the air-flow and SARS-CoV-2 Coronavirus build-up in a restaurant in Guangzhou, China [25, 23]. It is concluded that the outbreak of Covid-19 pandemic in the restaurant in January 2020, is due to the build-up of the airborne droplets and aerosols carrying the SARS-CoV-2 Coronavirus and would not have been prevented by standard ventilation. A comparison with standard models for aerosol concentration shows that, in the absence of ventilation, the decay of the aerosol concentration is also controlled by the decay time of the virions in aerosols. This decay time is very long, with low relative humidity, and a steady state is not achieved in the time-frame of the contagion. Instead the concentration exhibits a polynomial increase and reaches infectious levels in a relatively short time, explaining the outbreak in the restaurant in Guangzhou.
\end{abstract}

\section{Introduction}

We develop a model describing the concentrations of small droplets and aerosols as a function of time. The small droplets quickly evaporate into aerosols and we show that the aerosol concentration may increase linearly in time, with the reinjection of the contaminated air, and not quickly approach a steady state concentration, as predicted by the conventional models.

The understanding of the mechanism of infection by the SARS-CoV-2 Coronavirus has evolved considerably since the beginning of the Covid-19 pandemic. It is believed that the most common form of transmission is by respiratory droplets containing the coronavirus. These droplets were classically divided into heavier droplets that settle at a limited distance around the infected individual, small droplets, 5-10 micrometers in diameter, and aerosols (or droplet nuclei) $<5$ 
micrometers in diameter, that can remain airborn for an extended period and are convected by air inside confined spaces. The more recent scientific understanding is more nuanced. The pathogencontaining droplet can be carried by a turbulent cloud, emitted when the infected person coughs or sneezes. The droplets are active particles in this turbulent cloud and interact with the turbulent air and with each other. Experiments show that the cloud can rapidly spread up to a distance of 7-8 meters [7, 31, 9] (up to 25 feet). The droplet cloud, that is carried by air and can be airborne for hours, overlaps the artificial boundary of droplets and aerosols. We will adopt the terminology droplet/aerosols, in this paper, for droplets and aerosols around 1-10 micrometers in diameter. This term will apply to all droplets and aerosols that are airborne for times greater than $1-10 \mathrm{~min}-$ utes, that carry the coronavirus and are a source of contagion. The small droplets evaporate quickly and turn into aerosols as they are transported by air and interact with the ventilation system, so if we exclude large drops and droplets $>10$ micrometers, we will be able to restrict our conclusions to the concentration of aerosols $<5$ micrometers in diameter.

There is mounting evidence that droplet/aerosols are an important source of contagion, in confined spaces, see [28, 27, 8, 29, 11, 20, 22]. This makes it important to establish models that allow a computation of the concentration of the droplet/aerosols and how that concentration evolves over time. Such models would allow a computation of the risk of contagion and develop methods for its mitigation. In this paper we develop such a model based on Lagrangian fluid dynamics of turbulent air-flow. Then we use it to analyze an outbreak of infection [25] in a restaurant in Guangzhou, China, and compare with a numerical and experimental study [23] of the same outbreak. We develop a model describing the concentrations of droplet/aerosols as a function of time. Since these small droplets quickly evaporate into aerosols, we show that the aerosol concentration may increase linearly in time with re-injection of the contaminated air, and not quickly approach a steady state concentration, as predicted by the conventional models. The reason for this is that with poor or non-existent ventilation only the decay of the virions in aerosols controls the decay of concentration. This virion decay time is very long [34] and [36], with low relative humidity. The Lagrangian theory describes the distribution of the aerosols in space and permits a computation of parameters in this model.

Our study shows that the concentration of the aerosols may increase significantly in a confined space in the span of one hour. This increase in concentration is the likely cause of the outbreak in the restaurant in Guangzhou, China, see [25] and [23]. As discussed in [30] this increased concentration may spread by air-conditioning ducts from one confined space to another. The remedy is to greatly increase the effectiveness of the air-conditioning filters to filter microscopic particles, see [30] and Appendix D, in the supplementary information, increase the relative humidity, and/or create a cross flow through the confined space that washes the droplet/aerosols away. Applying such methods in confined spaces such as homes, restaurants, offices, buses and classrooms, may dramatically decrease the contagion rates. Their application may be crucial in controlling the final wave of Covid-19 infections before the pandemic can be suppressed by vaccinations.

\section{Application to Covid-19}

In a recent paper [25] the infection of 3 families by one infected person in an air-conditioned restaurant in Guangzhou, China, is described, see Figure 1. One of the families had travelled from Wuhan and ate lunch in the restaurant where the other two families were present. The first family 


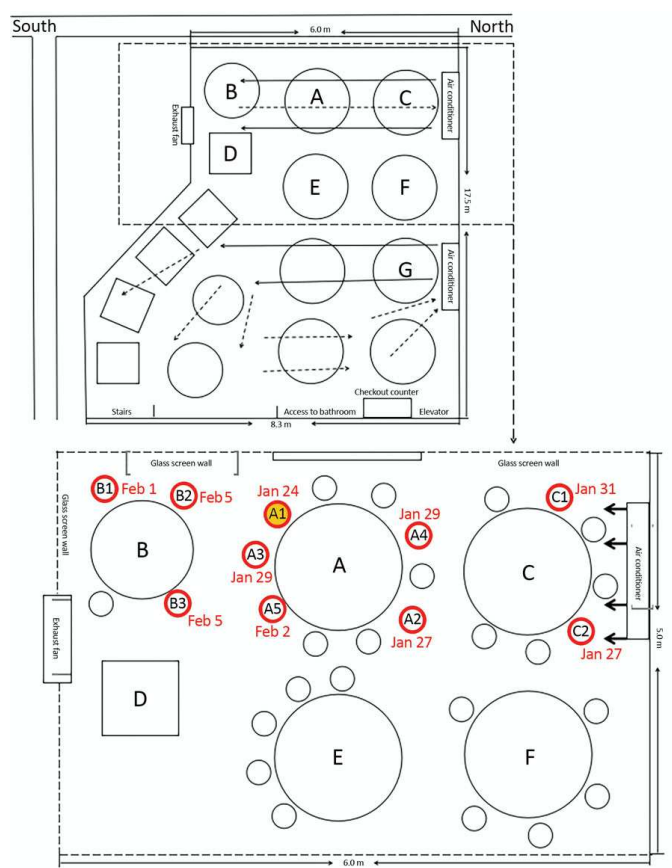

Figure 1: A sketch showing the arrangement of restaurant tables and air conditioning airflow at the site of an outbreak of 2019 coronavirus, in Guangzhou, China, 2020. Red circles indicate where patients that developed the disease were located; yellow-filled red circle (A1) indicates the location of the infected patient. The figure is taken from [25].

contained one person (A1) who fell ill later the same day and went to the hospital. 12 days later 9 other members of the three families had fallen ill with Covid-19. The infection is consistent with droplet transmission because no one else at the restaurant nor the servants fell ill. Only the persons in the direct airstream of the air-conditioner fell ill.

In the following analysis we will use the above theory to simulate the dispersion of the droplet/ aerosols in the restaurant. The part of the restaurant where the contagion took place is of dimensions 6 meters length and 3 meters width, see Figure 1 . Air-conditioners are set so that the restaurant guest experience wind air blowing at the velocity of 0.25 meters per second, see [1]. The corresponding Taylor-Reynolds number is $R e_{\lambda}=705$, since the air is flowing along a 3 meter distance from Table A to the wall where the air-conditioner is located. The three meter distance, perpendicular to the air-flow, is taken to be the distance across the largest table, where the infected person was sitting, and including one half-meter for each person sitting on opposite sites. Using the energy dissipation $\varepsilon=1.2$, taken from the experimental and simulation data in [2], we get the Kolmogorov time scale $\tau_{\eta}=3.55 \mathrm{~ms}$ (milliseconds). We employ the theoretical structure function $S_{2}$ in Appendix A, in the supplementary information, and use the stochastic closure theory (SCT), see [3, 4, 5], to compute the coefficient $C_{2}$ in the exponential of the formula for the Richardson distribution (B.1) in Appendix B, in the supplementary information. This computation, that interpolates the coefficients computed in [19] for the Reynolds number $R e_{\lambda}=705$, is explained in Appendix $\mathrm{C}$, in the supplementary information.

The results of the simulations for the restaurant in Guangzhou are shown in Figures 2-6. In Figure 2 we show the Richardson distribution as a function of time and space. We assume that 

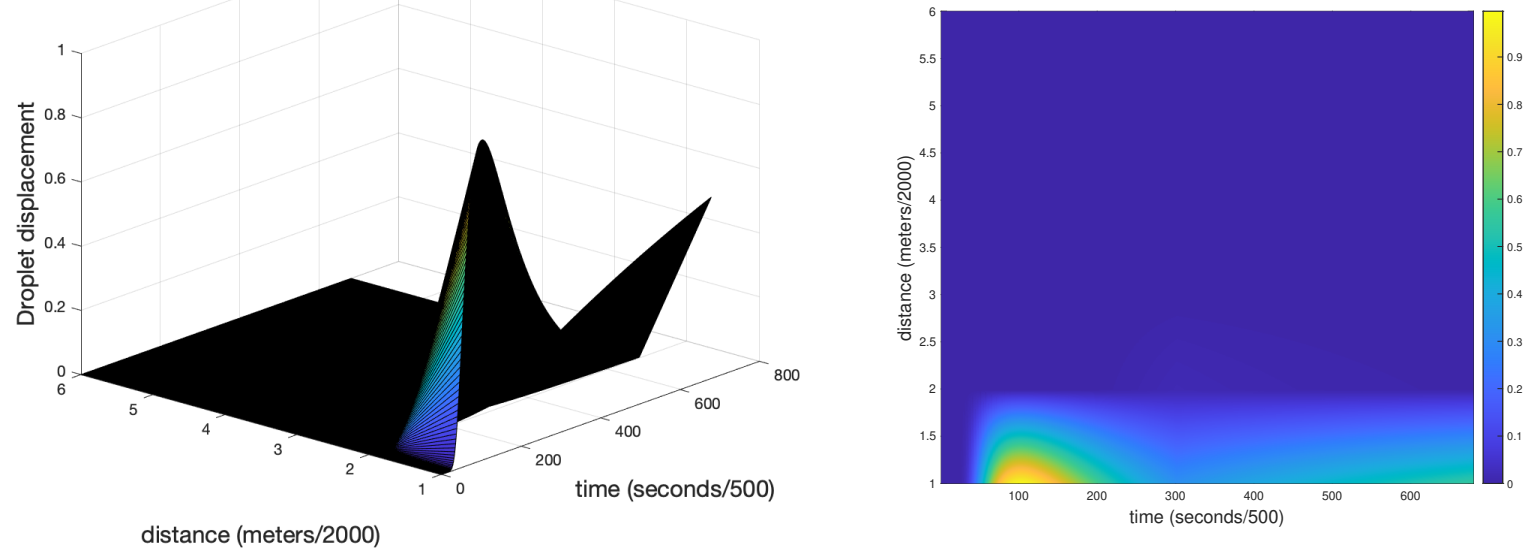

Figure 2: The Richardson distribution for the particle separation.

the infected person exhales 12 times a minute and each exhale event last 2.4 seconds, or almost half of the breathing period, that is 5 seconds. We are taking twice the lower limit of the breathing rate, that we take to be the shortest time of a breathing event, or the most forceful one (a cough), see [14]. Each time unit is $\tau_{\eta}=3.55 \mathrm{~ms}$, and we are computing 2000 units for a total of 7.1 seconds. In the left figure in Figure 3, we show the complete distribution for one exhaling event, roughly 340 points $\approx 1.2$ second. Observe that the distance between the points is greater in the beginning (Richardson dispersion) but becomes smaller after the second half, 1.2 to 2.4 seconds, of the exhale event (vortex-streaching diffusion). We make this clearer in Figure 1, in Appendix $\mathrm{A}$, in the supplementary information, where on the left we plot the distribution of the cloud for first 340 points or 1.2 seconds and on the right for the subsequent 340 point or the subsequent 1.2 seconds, to see what happens to the initial cloud immediately afterwards. We call the left the vortex-building part and the right the vortex-streaching part of the exhale and propagation events, because the first involves vortex-building and the second vortex-streaching eddies. The cloud is clearly more diffuse during the vortex-building part (Richardson dispersion) and more concentrated during the vortex-streaching part. The concentration of the droplet/aerosols is slightly (1.16 times) greater during the vortex-streaching phase. The cloud is traveling with the airflow, at $0.25 \mathrm{~m} / \mathrm{s}$, so the total length of the cloud is 0.6 meters, the vortex-building part extends to 0.3 meters and the vortex-streaching part covers the subsequent 0.3 meters.

The Richardson distribution for the particle separation for passive scalars, in Appendix B, in the supplementary information, allows us to compute the shape and the volume of the cloud of droplet/aerosols exhaled by an infectious person. We assume that the person is stationary (seated) and that he talks to the people around him and breathes in various directions in an $180^{\circ}$ angle with him in the center. The velocity of is exhaled breath is taken to be 1.5 meters per second and this is what creates the Lagrangian cloud, or the vortex-building eddies. This velocity is not sustained because the infected person both exhales and inhales. The cloud extends in the radial $(y)$ direction but it is carried by the ambient air flow, that is driven by the poorly ventilated air conditioning, in a fixed direction, at the velocity of 0.25 meters per second. Thus no matter in which direction the puffs of droplet/aerosols were exhaled, they get shaped into a cloud with a cylindrical symmetry 

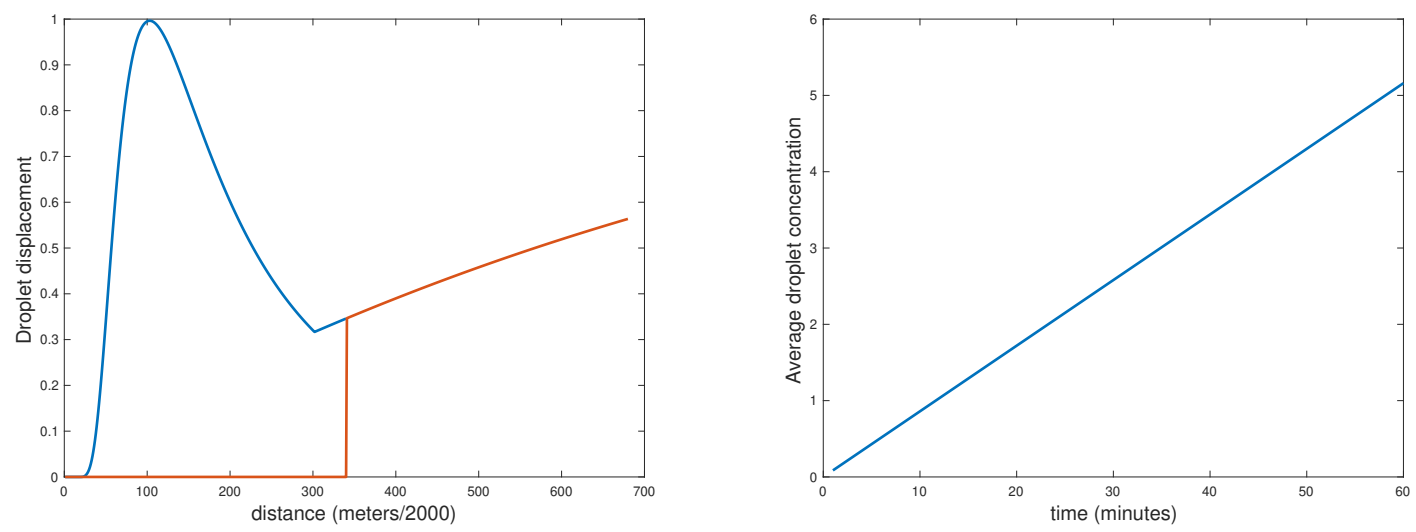

Figure 3: (Left) The total distribution of droplet displacement after 2.4 second of exhaling. The blue (vortex-building) part corresponds to the first 1.2 second of the exhale event and the red (vortex-streaching) part the subsequent 1.2 seconds. This is a cross-cut giving the longitudinal shape of the, cylindrically symmetric, exhaled cloud. (Right) The average, in space (inside the pyramid), droplet concentration as a function of time, in minutes, normalized by the concentration at the infected person. The concentration builds up to one (the concentration at the infected person) in 12 minutes and 5.16 times that in an hour.

by the ventilation wind along the axis $(x)$, see Figure 5, and the exhalation velocity along the radial (y) direction. We are assuming the turbulent eddies are created by the exhalation but afterwards they evolve by the turbulent Lagrangian flow. This assumption is reasonable but should be tested in future simulations and experiments. Using the Richardson distribution we can compute the shape of the cloud in the radial (y) direction and using the velocity of the ambient air flow, in the $x$ direction, we compute the extent of the cloud in the $x$ direction. The infectious person is sitting with his head in the center of the middle of the cylinder in Figure 5. This geometry allows us to compute the emission rate of the droplet/aerosols below.

It is important to understand that the Richardson distribution is not giving the instantaneous shape of an exhaled cloud. The exact shape of the exhaled cloud will vary from one exhale event to another, a reflection of the fact that these are stochastic processes. But a large number of these events are taking place, 12 per minute, and the Richardson distribution is producing the shape of the averaged cloud, averaged over all of these exhale events. Moreover, a person does not get infected by small droplet and aerosols from a single exhale event. Infection results from an exposure to a large number of exhale events over time, see [17]. Thus the Richardson distribution is exactly giving us the information that we need.

One can make a rough estimate of how the concentration of the droplet/aerosols builds up in time using the above theory and the CFD (computational fluid dynamics) simulations shown in Figure 4. The volume of the whole space containing the contamination is 3 by 6 by 3.14 meters $=56.52 \mathrm{~m}^{3}$, see [23]. A pyramid with base on the wall opposite the air-conditioner mostly contains the blue contamination cloud, see Figure 4. Thus the cloud does not fill the available space but only this pyramid with the volume $56.52 / 3=18.84 \mathrm{~m}^{3}$. The radius of a cylinder containing the exhaled cloud, is the height of the cloud, and reaches its maximum at 100 units on the left figure in Figure 3. This corresponds to 0.35 seconds and the velocity determining the spread is 
probably not the velocity at which the cloud is drifting but the velocity at which the dropletsaerosols are being ejected. This is taken to be $1.5 \mathrm{~m} / \mathrm{s}$ in [23] and we will use the same value here. Recall that there are two air velocities involved: the velocity of the exhaled air, in the radial $y$ direction, and the ambient wind velocity, in the $x$ direction, see Figure 5. This give the maximum radius 1.5 times $0.35=0.53$ meters. Computing the area under the curves (blue and red) in Figure 3 (left), and rotating it around the $x$ axis, we get the volume, $0.132 \mathrm{~m}^{3}$. The cloud has a vase shape, see Figure 3 (left), but its volume is equivalent to the volume of a cylinder of radius 0.264 meters and length 0.6 meters, see Figure 5. If we let the exhaled clouds be contained in a cylinder with this radius extending the length of the contamination area, we get the volume $1.32 \mathrm{~m}^{3}$ for this cylinder. Namely, the length of the cylinder, 6 meters, is 10 times the length of the exhaled cloud, 0.6 meters. The droplet/aerosols in the cylinder now get spread to the pyramid, by the airconditioning and heat conduction, see Figure 5. This decreases their concentration by a factor of $18.48 / 1.32=14$. However, the infected person keeps exhaling a new droplet/aerosol cloud every 5 seconds and since the extent of these clouds is 0.6 meters he needs to exhale 10 clouds to fill the 6 meter long cylinder. This takes 50 seconds or 0.83 minutes. In one hour he has filled the cylinder $72=60 / 0.83$ times. Thus with no droplet/aerosols lost, the exhaust fan was closed see [23], the resulting concentration in the pyramid is $72 \times 1 / 14=5.16$ times what it was in the cylinder, the first time it was full. In other words, the concentration for everyone sitting at the three tables A, B and C, in Figure 1, in less than 12 minutes, is what it would be if they were sitting next to the infected person. In one hour, the concentration is more than five times what it was initially, if every person on the three table was sitting next to the infected person. This build-up of the concentration of the droplet/aerosols containing the SARS-CoV-2 Coronavirus is the likely reason for the outbreak in the restaurant in Guangzhou.

\section{The Concentration of Droplets, Aerosols and Virions}

The conventional way of modeling the concentration of droplets and aerosols in a room is by a differential equation

$$
\frac{d}{d t} C=E / V_{I}-\lambda C
$$

where $\mathrm{E}$ is the exhalation rate, in dimensions droplets and aerosols per volume per minute, and $V_{I}$ is the volume of infectious air. In the example of the restaurant above $V_{I}$ is the volume of the pyramid and not the volume of the whole airspace. This means that the frequently made hypothesis, that the whole available air space is well mixed, does not hold for the restaurant and may not be true in other cases. $\lambda$ is the decay coefficient of the concentrations, it is composed of a sum of three decay rates $\lambda=k_{v}+k_{s}+k_{d}$, where $k_{v}=1 / t_{v}, k_{s}=1 / t_{s}$, and $k_{d}=1 / t_{d} . t_{v}$ is the time it takes to ventilate the room, $t_{s}$ is the settling time of the droplet/aerosols and $t_{d}$ the decay time of the viruses in the aerosols.

The vents in the restaurant were closed so there was no ventilation and we can take $k_{v}=0$. (We elaborate on this choice in the Discussion Section.) The droplets settle over time, but they were being blown around by the air conditioner and quickly evaporate into aerosols. This process was presumably helped by modest heating. The estimates of the settling time range from 8-14 minutes, see [33] and [32], whereas the evaporation of the droplets only takes a few seconds, see [18] and [33]. Thus the settling time is just relevant for the process of turning droplets into aerosols. The 


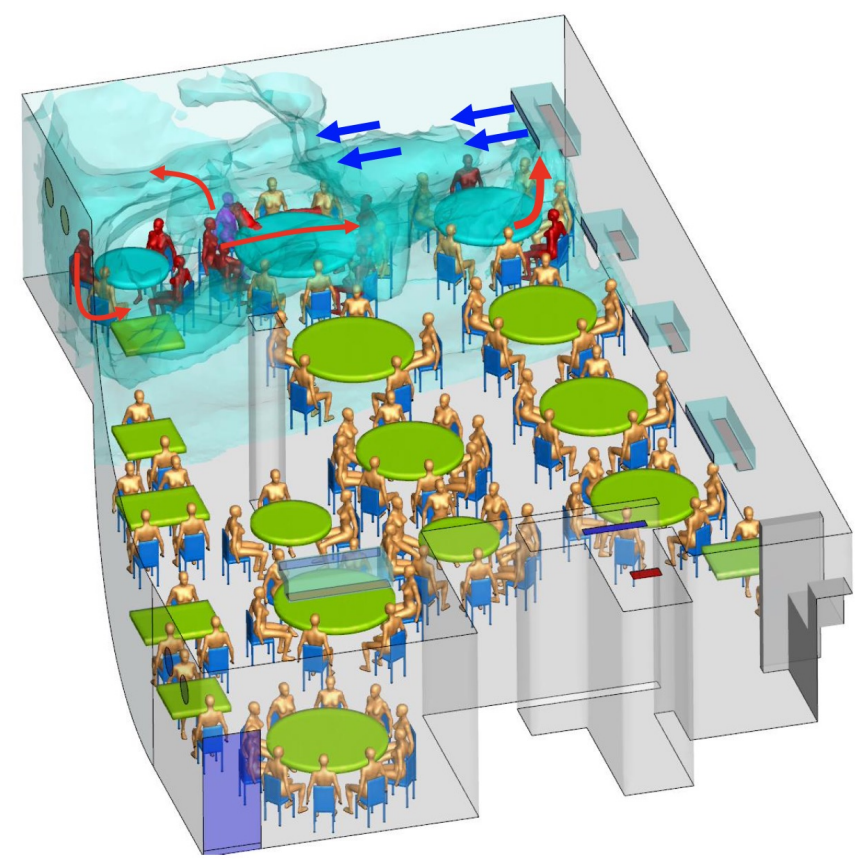

Figure 4: A CFD (computational fluid dynamics) simulation of the contagion in the restaurant in Guangzhou China. The blue gas models the contamination by the droplet/aerosols. The infected people are colored red. Notice that the contaminated region roughly forms a pyramid with base on the wall opposite to the air-conditioner. The figure is take from [23].

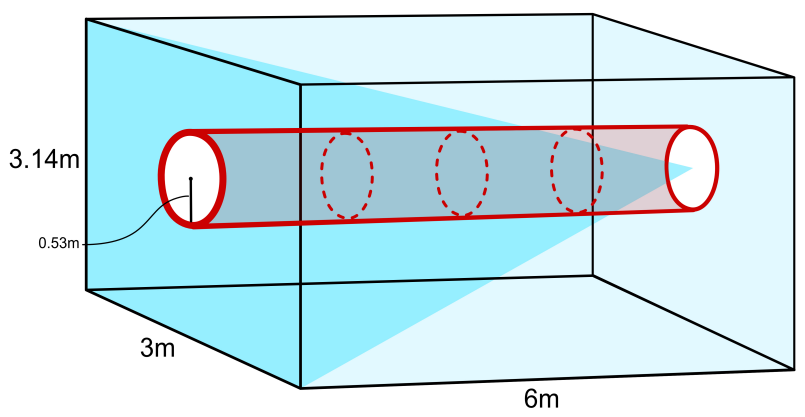

Figure 5: A cartoon (not to scale) of the volume (red cylinder) where the droplet/aerosol clouds propagate and the volume (blue pyramid) that the droplet/aerosol clouds get spread to by the airconditioning. 

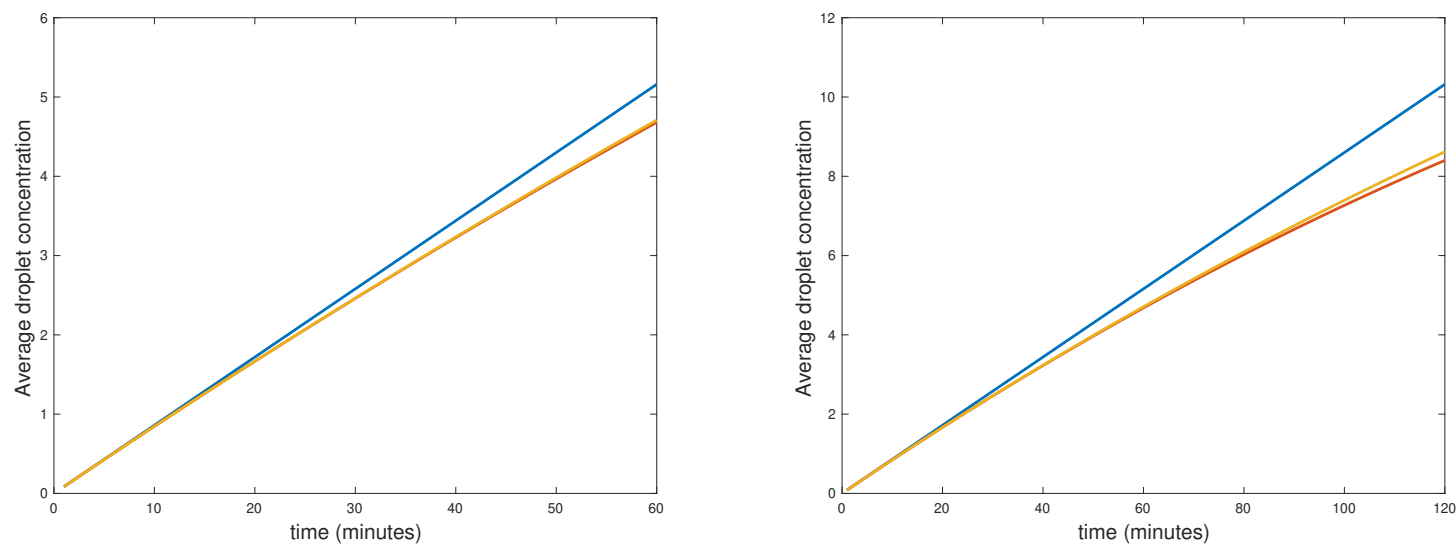

Figure 6: (Left) The aerosol concentration for one hour, top (blue) linear curve, middle (orange) solution of the ODE, bottom (red) the quadratic polynomial. The last two lines are hardly distinguishable. (Right) The aerosol concentration for two hours, top (blue) linear curve, middle (orange) solution of the ODE, bottom (red) the quadratic polynomial. The last two lines begin to separate on the right.

settling of the aerosols is negligible, even if they settle on the tables they are being blown around by the airflow, and by restricting $C$ to be the concentration of aerosols, including the nuclei of the evaporated droplets, we can take $k_{s}=0$. This leaves the decay time of the virions in aerosols. This time is estimated experimentally in [34], [16] and [12]. It depends on the relative humidity and is similar to that of Influenza A. Since the dependence of the latter on the relative humidity is known, we will use the decay coefficients for Influenza A [36] as a proxy. The relative humidity in Guangzhou in January is $72 \%$ but weather is cold and the relative humidity in a heated restaurant can be expected to be much less. We pick it to be $21 \%$ and use the corresponding value $k_{d}=0.0031$ from [36]. Higher values will give qualitatively similar theory, but slightly different quantitatively, see Appendix D, in the supplementary information. The aerosol concentrations now lies between the two polynomials

$$
\frac{E}{V_{I}}\left(t-k_{d} t^{2} / 2\right) \leq C(t) \leq \frac{E}{V_{I}} t,
$$

where $\frac{E}{V_{I}}=0.086=5.16 / 60$ is the exhalation rate per minute divided by the volume of the contaminated space. It continues to increase through the time interval and reaches its maximum $C(60)=4.72$ at the end of the hour, see Figure 6

If we use the $65 \%$ relative humidity in [34] and their corresponding measured value of $k_{d}=$ 0.01 , the picture does not change much, $C(t)$ continues to increase through the hour and reaches its maximum $C(60)=2.73$ at the end of it, see Appendix D, in the supplementary information. However, this relative humidity is too high for typical indoor humidity, 30\%-40\%, in winter.

We now discuss what the concentrations of virions are represented by the aerosol concentration $C=1$ at the infectious person, or in his or her exhaled cloud. It was shown above that the volume of the exhaled cloud after an 2.4 second exhale event is 1,320 liters. The tidal volume or the volume of the exhaled breath is 0.5 liters. Thus the concentrations of the droplets and aerosols decreases by a multiplicative factor of $0.5 / 1320=3.79 \times 10^{-4}$. Now the relevant unit is $T C I D_{50}$ or $50 \%$ tissue-culture infectious dose. The concentration of $T C I D_{50}$ in the respiratory track is $10^{5} T C I D_{50}$ 
per milliliter, see [34]. That gives $10^{8} T C I D_{50}$ per liter and multiplying with the above factor, we get the concentration $3.79 \times 10^{4} T C I D_{50}$ per liter in the exhaled cloud. It is not yet known what the infectious dose is for people, it is higher than for tissue culture, is but an inhale event, based on the tidal volume 0.5 liters, will involve $1.90 \times 10^{4}$ TCID $_{50}$ and if the effectiveness, of the infection, reduces the effective $T C I D_{50}$ by a factor of ten (this factor of ten is arbitrary and is just set to make the connection with the consensus of 1000 virions infectious dose, that still remains to be verified), then there is some consensus in the literature, see [26, 6, 12, 13, 35], that 1900 or roughly 2000, TCID 50 with 50\% infectious dose, is enough for infection. We can think about this as 1000 effective virions and this is what we normalize to be one, or the concentrations experienced by sitting next to the infected person, during the first couple of minutes of the outbreak event. Notice, that the concentration at the infected person may increase with time, so the normalization must be made with an average over initial time interval of one to two minutes. In one minute a person sitting next to the infected person will have inhaled 1000 effective virions, 12 times. Consequent, people sitting next to an infectious person for a sufficiently long time (10-15 minutes) are invariably infected.

The analysis above also makes it clear that if the concentrations are higher that one, it will take a shorter time for a person in the contaminated space to become infected. In fact, the higher the concentrations, the shorter the infection time.

\section{Discussion}

In [25] it was speculated that the flow generated by the air-conditioning was blowing large droplets from table to table. This is highly unlikely since the air-conditioning flow $(0.25 \mathrm{~m} / \mathrm{s})$ does not have enough energy to send the droplets on a ballistic trajectory between the tables, see [18]. In [23] the blame for the outbreak was put on the poor air-conditioning in the restaurant, caused by the closed vents and small injection of fresh air. We redid the simulation with the recommended [1] injection of fresh air 8 liters per second for each person in the infected area, to test this hypothesis. There are 21 persons at the three tables $\mathrm{A}, \mathrm{B}$ and $\mathrm{C}$ and during the 0.83 minutes it takes the infected person to fill the cylinder above with a droplet/aerosol cloud, 8,366 liters or $8.37 \mathrm{~m}^{3}$ are injected into the infected area, if the air-conditioning follows recommendations. Adding this to the total volume above we get $63.81 \mathrm{~m}^{3}, 21.27 \mathrm{~m}^{3}$ of which pass through the pyramid containing the infected air. This amounts to adding $3.36 \mathrm{~m}^{3}$ per minute, to the volume $18.48 \mathrm{~m}^{2}$ of the contaminated pyramid in Figure 5. In one hour we add $201.6 \mathrm{~m}^{3}$ of fresh air to the pyramid, for an air change per hour $(\mathrm{ACH})$ equal to 10.70. This is high due to the high density of the people in the restaurant. This gives the damping coefficient $k_{v}=0.178$ in Equation 3.1. The concentration now quickly increases and is 0.5 , see Appendix D, in the supplementary information, in one hour. This is still high enough to cause an infection. Thus fixing the air-conditioning, significantly decreases the concentration but does not completely solve the problem. We also asked the question how much injection of fresh air would we need to make the restaurant safe? If we increase the ACH to 52.2, then $k_{v}=0.87$ and this decreases the concentration to 0.1 in one hour. In other words we need more than 5 times the recommended air injection to make the contaminated pyramid have (a safe) concentration less than 0.1 in one hour, see Appendix D, in the supplementary information. This amounts to a brisk breeze blowing through the restaurant.

It is clear from the above discussion that the part of the restaurant where the contagion took 
place was a particularly vulnerable place for transmission by aerosols. The air flow from the air-conditioner was restricting the space that needed to be ventilated, to the pyramid in Figure 5 , not the whole space in the back of the restaurant. The vents were closed so there was not fresh air coming in, the gentle heating accelerated the evaporation of small drops into aerosols and the density of the patrons was very high. These factors conspired to make the ventilation coefficient $k_{v}=0$ in Equation 3.1. The settling coefficient of the aerosols, that were being blown around and reinjected into the pyramid by the air-conditioner, was also $k_{s}=0$. The only mitigating influence was the decay coefficient of the aerosols, that is very small $k_{d}<<1$, since relative humidity was probably low. The combination of these factors made the back of the restaurant in Guangzhou a very infectious place in a short time.

If the air flow from the air-conditioner was much stronger, than we have assumed, then it may have carried some small droplets containing virions from Table A to Table C, see Figure 1. Indeed this is what happened in a resent study of a restaurant in South Korea where the air-conditioner was blowing particularly hard, see [21]. If the air-conditioner in the restaurant in Guangzhou was blowing very hard, this could explain how the persons at Table $\mathrm{C}$, downstream from the infected person at Table A, got infected, see Figure 1. But it cannot explain how the persons at Table $\mathrm{B}$, upstream from the infected person, got infected. The only possible explanation is that the concentration of the aerosols at Table B was sufficiently high to cause an infection. The small droplets would all have been aerosolized going through the air-conditioner and carried by the air flow along the ceiling, to descend along along the wall, opposite the air-conditioner, to Table B. The aerosols must have accumulated at Table B as in the rest of the contaminated pyramid, see 5 , because low concentrations would not have caused the same number of infections.

In [30] the transmission of the virus droplet/aerosol through an air-conditioning system is discussed with the conclusion that greater volume of outdoor air and MERV-13 or HEPA filters with the capacity of filtering our the droplet/aerosols need to be used. This is consistent with our observations. However, the air-conditioners in current use may be unable to use MERV-13 filters or handle the required volume of outdoor air. Indeed a new generation of air-conditioners that meet these requirements may be needed.

The Lagrangian method used in the paper has a clear advantage over the full CFD (computational fluid dynamics) simulation. The latter is very expensive (in computer time) and timeconsuming, whereas the Lagrangian method gives the average over many CFD simulations. The Lagrangian method only requires knowledge of the dimensions of the space and the wind velocity, and can be run on a standard desktop or laptop computer. The CFD has some advantages, it gives the shape of the contaminates space, the pyramid in Figure 5, showing the well-mixed hypothesis does not hold in the restaurant, and produces the Lagrangian results if many simulations are performed and their averaged computed. The geometry of the pyramid can however also be obtained from the Lagrangian analysis with slightly more work. The great advantages of the Lagrangian analysis is that it gives the distribution (concentration) of the aerosols in the contaminated area and then simple ODEs (ordinary differential equations) can be solved to determine its infectiousness.

\subsection{Some problems with previous analysis}

In this paper we have shown that the aerosol concentration in the restaurant in Guangzhou could increase as a second order polynomial in time, reaching the concentration $C=4.72$ times what it was, $C=1$, next to the infected person for the first couple of minutes of the outbreak event. So 
why has this not been discovered by previous studies? The reasons seem to be that the previous studies were based on faulty assumptions about the conventional model, the most serious of which are the assumptions of a steady state and the assumption of a well-mixed air, see [24, 10, 15].

We discussed above how the airflow could not carry the larger droplets ballistically between the tables as speculated in [25], however the airflow can easily carry the much lighter aerosols and continue to blow them around the contaminated pyramid. There is every reason to believe that the aerosols were sucked up by the air-conditioner and distributed to the back wall were they infected the patrons at Table B, see Figure 1, since the air-conditioner did not have a filter capable of filtering out the aerosols. In [25] smear samples were collected from the air-conditioner and since those were negative it has lead to the conclusion that aerosols could not have been distributed by the air-conditioner. This reasoning is problematic, a positive result from smear samples would have confirmed the distribution of aerosols but a negative result does not show that no distribution took place. In fact, a spread of aerosols by the air-conditioner to table B is the only logical explanation of how the three people at table B got infected. The aerosols collecting on surface of the airconditioner where probably short lived and the airflow had plenty of time to clean them all up in the days between the outbreak and when these samples were taken. Thus the negative result from the smear samples is expected.

In [23], the concentration of tracer gas was measured but averaged over time (assuming the existence of a steady state). The time averaged concentrations at Tables B and C, were found to be close to one, but this only shows that the relative concentrations at the three tables are similar over time. It obscures the fact that the (spatially averaged) concentration at all the tables is increasing over time and the relevant reference concentration that shows this, is the concentration at Table A, see Figure 1, during the first couple of minutes of the outbreak event, see Section 3. The CFD (computational fluid dynamics) simulation implemented in [23] show that the air in the contaminated part of the restaurant is not well mixed, but in spite of this, it assumed in [23] that the very weak airflow through elevator and fire door exhausted by the fan in the restroom, see Figure 4, is distributed throughout the restaurant. (In the experiment [23], desk fans were placed on the tables to aid the mixing.) It is very unlikely that any of this air made it to the back of the restaurant, given the strong transverse flow from all the air-conditioning units. Even, if the assumptions in [23] were correct the quantity of air ( $\mathrm{ACH}$ ) was so small that it would amount only to (be equivalent to) a small increase in humidity, see Appendix D, in the supplementary information. The growth of the concentration would not be affected much.

The final problem that seems to have mislead some studies is the assumption of the influence of settling time for some droplets, see [15]. As we discussed above the small droplets evaporate long before they settle and their evaporation is accelerated by heating of the air by the air-conditioner. The only exception is a very strong airflow such as in [21], in that case the settling time of the smaller droplets will play a role.

In the most infectious cases, there may be strong airflow, without any ventilation, that reinjects the aerosols into the confined space and prevents the air in the space from being well-mixed, thus increasing the aerosol concentration further. This seems to have been the case in the restaurant in Guangzhou. The concentration is such spaces can quickly increase to very infectious levels. 


\subsection{Limitations}

In our study we have excluded the large droplets $>10$ micrometers. Their inclusion would still be a Lagrangian problem but they are not passive scalars. The large droplets can influence the flow and their size and composition is also greatly influenced by the flow. One has to extend the theory to include these droplets and their dynamics. In principle this can be done with more work and the theory extended at least numerically, if not with theoretical formulas for the structure functions as in Appendix B, in the supplementary information.

Acknowledgements: The author wants to thank Klaus Schauser and Albert Oaten for enthusiastic support and Ken Beisser for expert advice on air-conditioning. He also wants to thank Luiza Angheluta for all her help and support and Knut Bauer for providing him with the graphic illustration in Figure 5 .

\section{References}

[1] 62.1, A. S. Ventilation for acceptable indoor air quality. 2019.

[2] Biferale, L., Bodenschatz, E., Cencini, M., Lanotte, A. S., Ouellette, N. T., TOSCHI, F., AND XU, H. Lagrangian structure functions in turbulence: A quantitative comparison between experiment and direct numerical simulation. Physics of Fluids 20, 6 (2008), 065103.

[3] BirniR, B. The Kolmogorov-Obukhov statistical theory of turbulence. J. Nonlinear Sci. (2013). DOI 10.1007/s00332-012-9164-z.

[4] BIRniR, B. The Kolmogorov-Obukhov Theory of Turbulence. Springer, New York, 2013.

[5] BirniR, B. From Wind-Blown Sand to Turbulence and Back. Springer International Publishing, Cham, 2016, pp. 15-27.

[6] Bordi, L., Piralla, A., Lalle, E., Giardina, F., Colavita, F., Tallarita, M., Sberna, G., Novazzi, F., Meschi, S., Castilletti, C., et Al. Rapid and sensitive detection of sars-cov-2 rna using the simplexa ${ }^{\mathrm{TM}}$ covid-19 direct assay. Journal of Clinical Virology (2020), 104416.

[7] Bourouiba, L. Turbulent gas clouds and respiratory pathogen emission, potential implications for reducing transmission of covid-19. JAMA Insights (2020).

[8] Bourouiba, L. Turbulent Gas Clouds and Respiratory Pathogen Emissions: Potential Implications for Reducing Transmission of COVID-19. JAMA 323, 18 (05 2020), 1837-1838.

[9] Bourouiba, L., Dehandschoewercker, E., and Bush, J. W. Violent expiratory events: on coughing and sneezing. Journal of Fluid Mechanics 745 (2014), 537-563.

[10] Buonanno, G., Morawska, L., And Stabile, L. Quantitative assessment of the risk of airborne transmission of sars-cov-2 infection: prospective and retrospective applications. medRxiv (2020). 
[11] Chen, W., Zhang, N., Wei, J., Yen, H.-L., And Li, Y. Short-range airborne route dominates exposure of respiratory infection during close contact. Building and Environment 176 (2020), 106859.

[12] Chia, P. Y., Coleman, K. K., Tan, Y. K., Ong, S. W. X., Gum, M., Lau, S. K., Sutjipto, S., Lee, P. H., Young, B. E., Milton, D. K., et Al. Detection of air and surface contamination by severe acute respiratory syndrome coronavirus 2 (sars-cov-2) in hospital rooms of infected patients. medRxiv (2020).

[13] Corman, V., Eckerle, I., Bleicker, T., Zaki, A., Landt, O., Eschbach-Bludau, M., van Boheemen, S., Gopal, R., Ballhause, M., Bestebroer, T., et Al. Detection of a novel human coronavirus by real-time reverse-transcription polymerase chain reaction. Eurosurveillance 17, 39 (2012), 20285.

[14] Dbouk, T., AND DRIKAKIS, D. On respiratory droplets and face masks. Physics of Fluids 32, 6 (2020), 063303.

[15] Evans, M. Avoiding covid-19: Aerosol guidelines. arXiv preprint arXiv:2005.10988 (2020).

[16] Fears, A. C., Klimstra, W. B., Duprex, P., Hartman, A., Weaver, S. C., Plante, K., Mirchandani, D., Plante, J., Aguilar, P. V., Fernandez, D., Et Al. Comparative dynamic aerosol efficiencies of three emergent coronaviruses and the unusual persistence of sars-cov-2 in aerosol suspensions. medRxiv (2020).

[17] for Disease Control, C., And Prevention. How COVID-19 Spreads. Accessed 28 August 2020 (Updated June 16, 2020).

[18] Holterman, H. Kinetics and evaporation of water drops in air, vol. 2012. Citeseer, 2003.

[19] Kaminsky, J., Birnir, B., Bewley, G., And Sinhuber, M. Reynolds number dependence of the structure functions in homogeneous turbulence. Journ. of Nonlin. Sci. (2020), $1-34$.

[20] Klompas, M., Baker, M. A., And Rhee, C. Airborne Transmission of SARS-CoV-2: Theoretical Considerations and Available Evidence. JAMA 324, 5 (08 2020), 441-442.

[21] Kwon, K.-S., Park, J.-I., Park, Y. J., Jung, D.-M., RYu, K.-W., And Lee, J.-H. Evidence of long-distance droplet transmission of sars-cov-2 by direct air flow in a restaurant in korea. Journal of Korean medical science 35, 46 (2020).

[22] Lednicky, J. A., Lauzard, M., Fan, Z. H., Jutla, A., Tilly, T. B., Gangwar, M., Usmani, M., Shankar, S. N., Mohamed, K., Eiguren-Fernandez, A., et al. Viable sars-cov-2 in the air of a hospital room with covid-19 patients. International Journal of Infectious Diseases 100 (2020), 476-482.

[23] Li, Y., Qian, H., Hang, J., Chen, X., Hong, L., Liang, P., Li, J., Xiao, S., Wei, J., LIU, L., ET AL. Evidence for probable aerosol transmission of sars-cov-2 in a poorly ventilated restaurant. medRxiv (2020). 
[24] LiU, Y. E. A. Aerodynamic analysis of sars-cov-2 in two wuhan hospitals. Nature (2020).

[25] Lu, J., Gu, J., Li, K., Xu, C., Su, W., Lai, Z., Zhou, D., Yu, C., Xu, B., And Yang, Z. Covid-19 outbreak associated with air conditioning in restaurant, guangzhou, china, 2020. Emerging Infectious Diseases 26, 7 (2020).

[26] Miller, S. L., Nazaroff, W. W., Jimenez, J. L., Boerstra, A., Buonanno, G., Dancer, S. J., Kurnitski, J., Marr, L. C., Morawska, L., And Noakes, C. Transmission of sars-cov-2 by inhalation of respiratory aerosol in the skagit valley chorale superspreading event. Indoor air (2020).

[27] Morawska, L., And Milton, D. K. It Is Time to Address Airborne Transmission of Coronavirus Disease 2019 (COVID-19). Clinical Infectious Diseases (07 2020). ciaa939.

[28] Morawska, L., Tang, J. W., Bahnfleth, W., Bluyssen, P. M., Boerstra, A., Buonanno, G., CaO, J., Dancer, S., Floto, A., Franchimon, F., et Al. How can airborne transmission of covid-19 indoors be minimised? Environment international 142 (2020), 105832.

[29] Qian, H., Miao, T., LiU, L., Zheng, X., Luo, D., AND Li, Y. Indoor transmission of sars-cov-2. Indoor Air n/a, n/a.

[30] REnAT MANASSYPOV PHD, P. Evaluating virus containment efficiency of air-handling systems. ASHRAE Journal 62, 7 (2020), 17-23.

[31] Scharfman, B., Techet, A., Bush, J., and Bourouiba, L. Visualization of sneeze ejecta: steps of fluid fragmentation leading to respiratory droplets. Experiments in Fluids 57, 2 (2016), 24.

[32] Somsen, G. A., van Rijn, C., Kooij, S., Bem, R. A., And Bonn, D. Small droplet aerosols in poorly ventilated spaces and sars-cov-2 transmission. The Lancet. Respiratory Medicine (2020).

[33] Stadnytskyi, V., Bax, C. E., Bax, A., And Anfinrud, P. The airborne lifetime of small speech droplets and their potential importance in sars-cov-2 transmission. Proceedings of the National Academy of Sciences 117, 22 (2020), 11875-11877.

[34] Van Doremalen, N., Bushmaker, T., Morris, D. H., Holbrook, M. G., Gamble, A., Williamson, B. N., Tamin, A., Harcourt, J. L., Thornburg, N. J., Gerber, S. I., ET AL. Aerosol and surface stability of sars-cov-2 as compared with sars-cov-1. New England Journal of Medicine 382, 16 (2020), 1564-1567.

[35] Watanabe, T., Bartrand, T. A., Weir, M. H., Omura, T., and HaAs, C. N. Development of a dose-response model for sars coronavirus. Risk Analysis: An International Journal 30, 7 (2010), 1129-1138.

[36] YAng, W., AND MARR, L. C. Dynamics of airborne influenza a viruses indoors and dependence on humidity. PloS one 6, 6 (2011), e21481. 
Figures

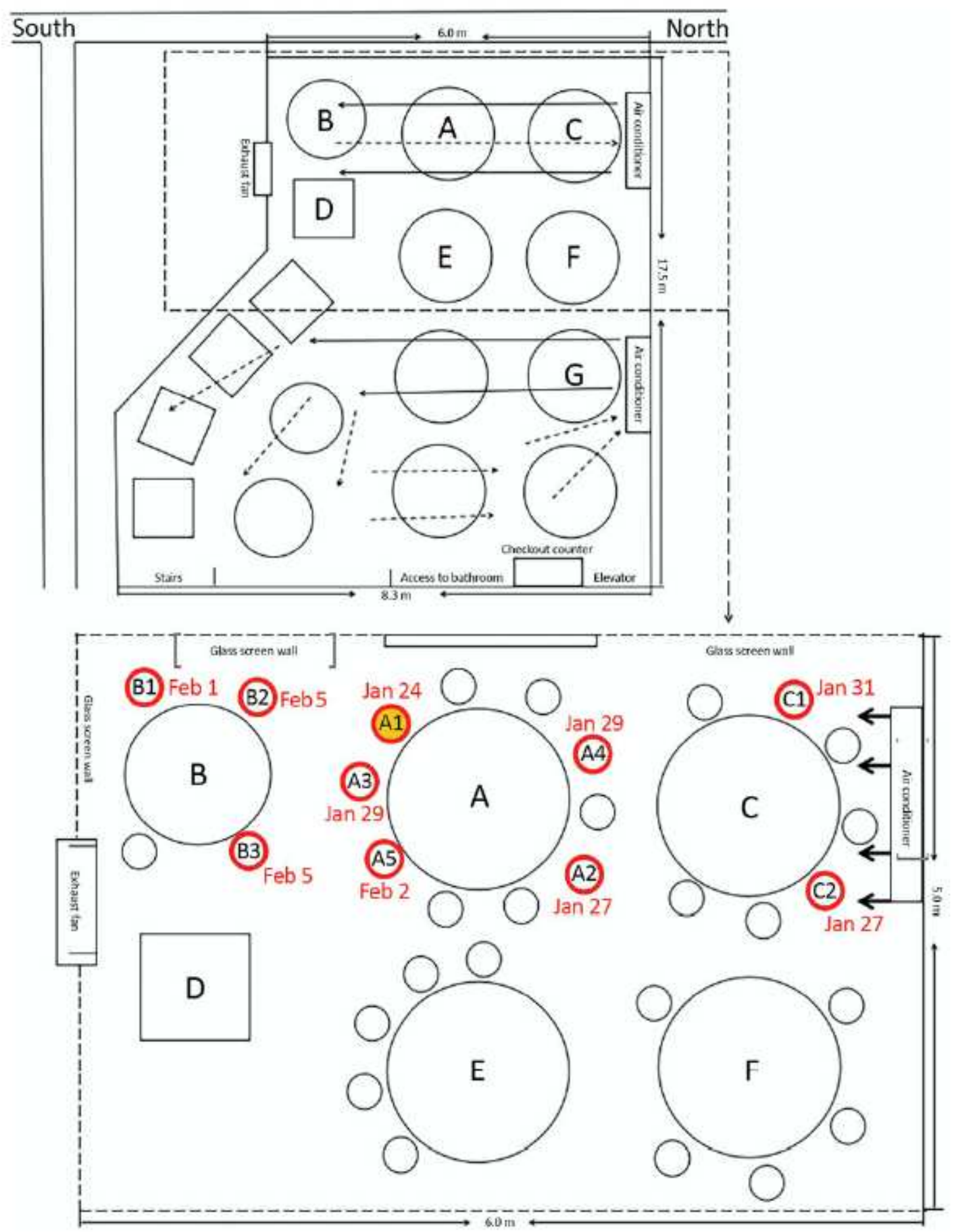

Figure 1

A sketch showing the arrangement of restaurant tables and air conditioning airflow at the site of an outbreak of 2019 coronavirus, in Guangzhou, China, 2020. Red circles indicate where patients that 
developed the disease were located; yellow-filled red circle (A1) indicates the location of the infected patient. The figure is taken from [25].
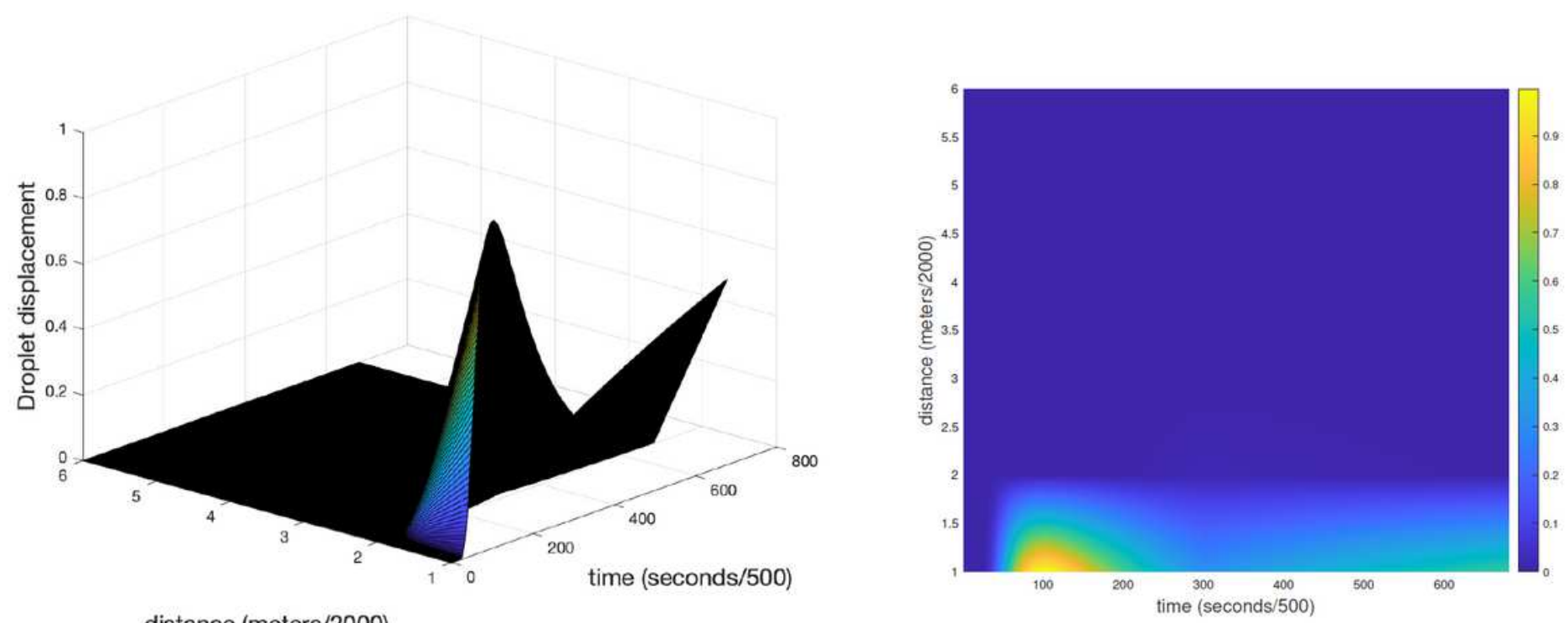

distance (meters/2000)

\section{Figure 2}

The Richardson distribution for the particle separation.
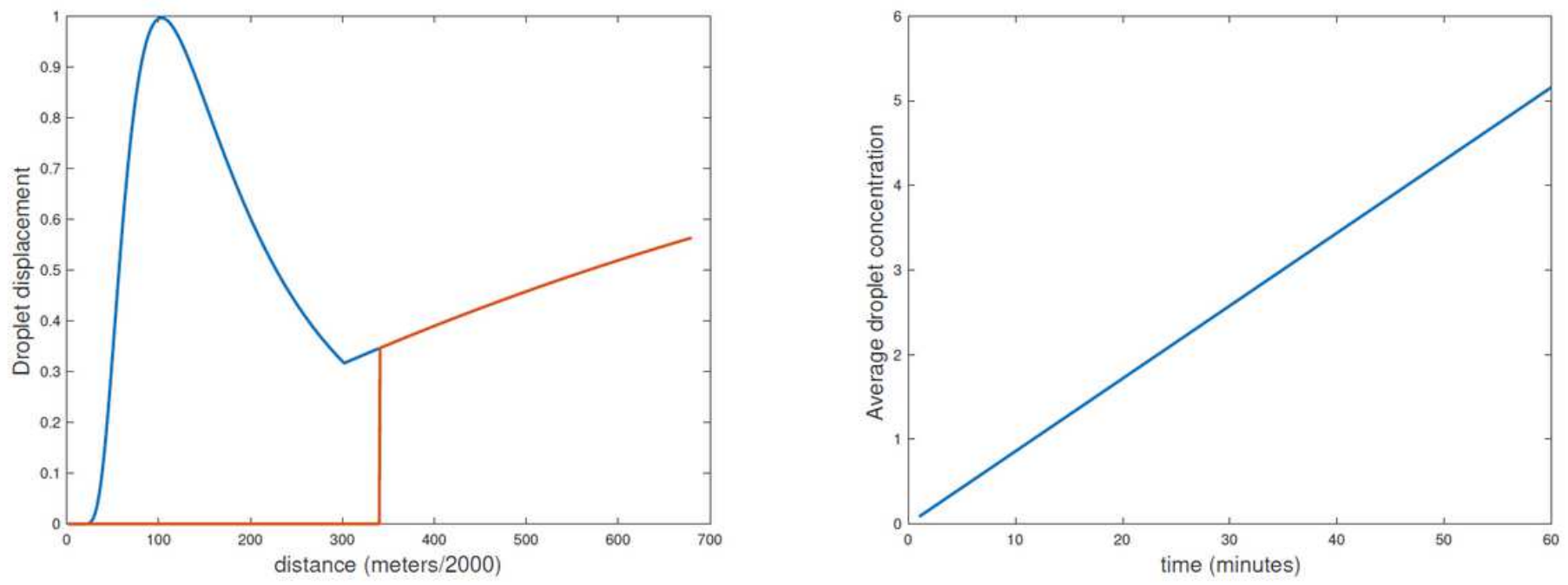

Figure 3

(Left) The total distribution of droplet displacement after 2.4 second of exhaling. The blue (vortexbuilding) part corresponds to the first 1.2 second of the exhale event and the red (vortex-streaching) part the subsequent 1.2 seconds. This is a cross-cut giving the longitudinal shape of the, cylindrically symmetric, exhaled cloud. (Right) The average, in space (inside the pyramid), droplet concentration as a function of time, in minutes, normalized by the concentration at the infected person. The concentration builds up to one (the concentration at the infected person) in 12 minutes and 5.16 times that in an hour. 


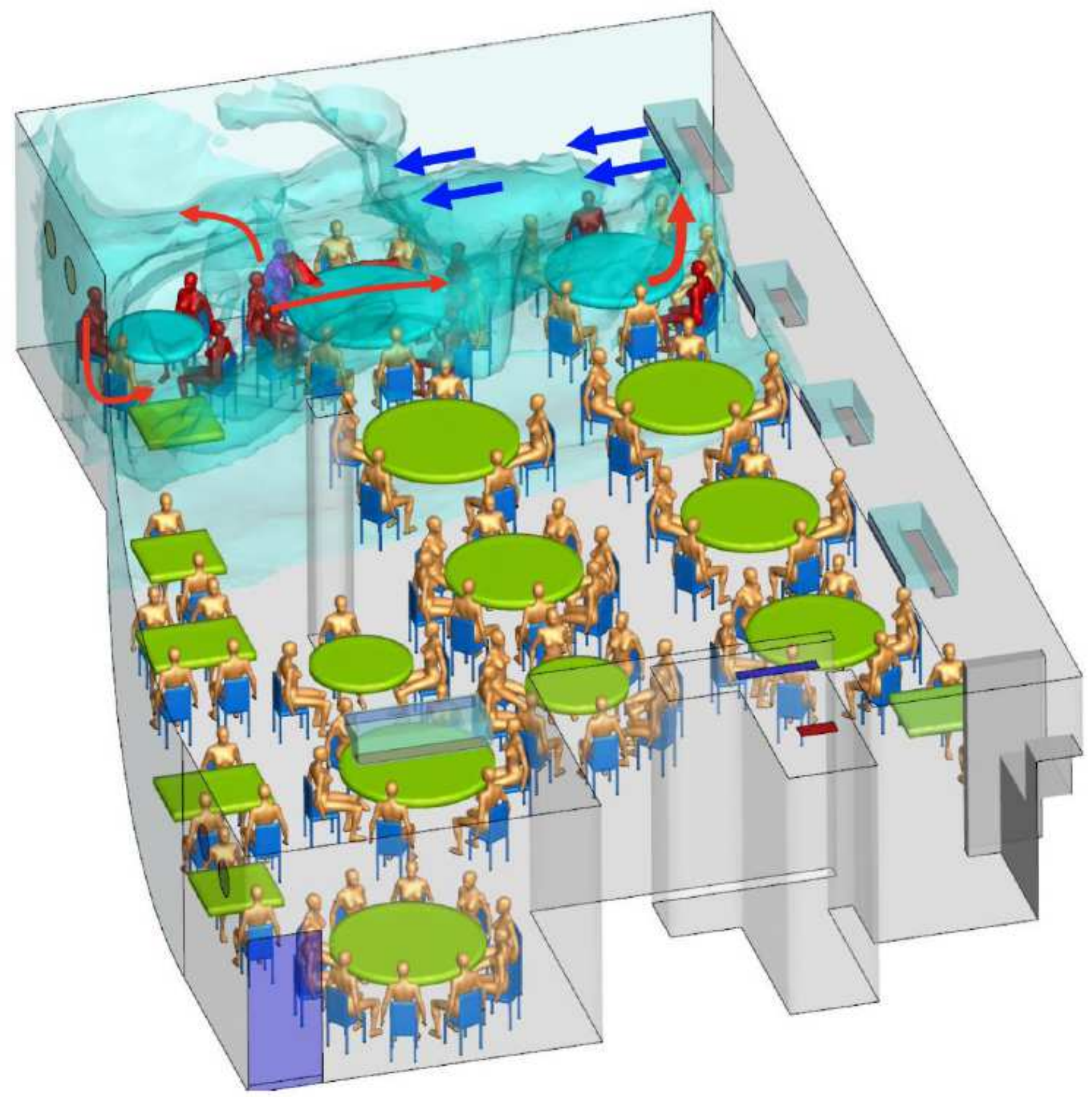

Figure 4

A CFD (computational fluid dynamics) simulation of the contagion in the restaurant in Guangzhou China. The blue gas models the contamination by the droplet/aerosols. The infected people are colored red. Notice that the contaminated region roughly forms a pyramid with base on the wall opposite to the airconditioner. The figure is take from [23]. 


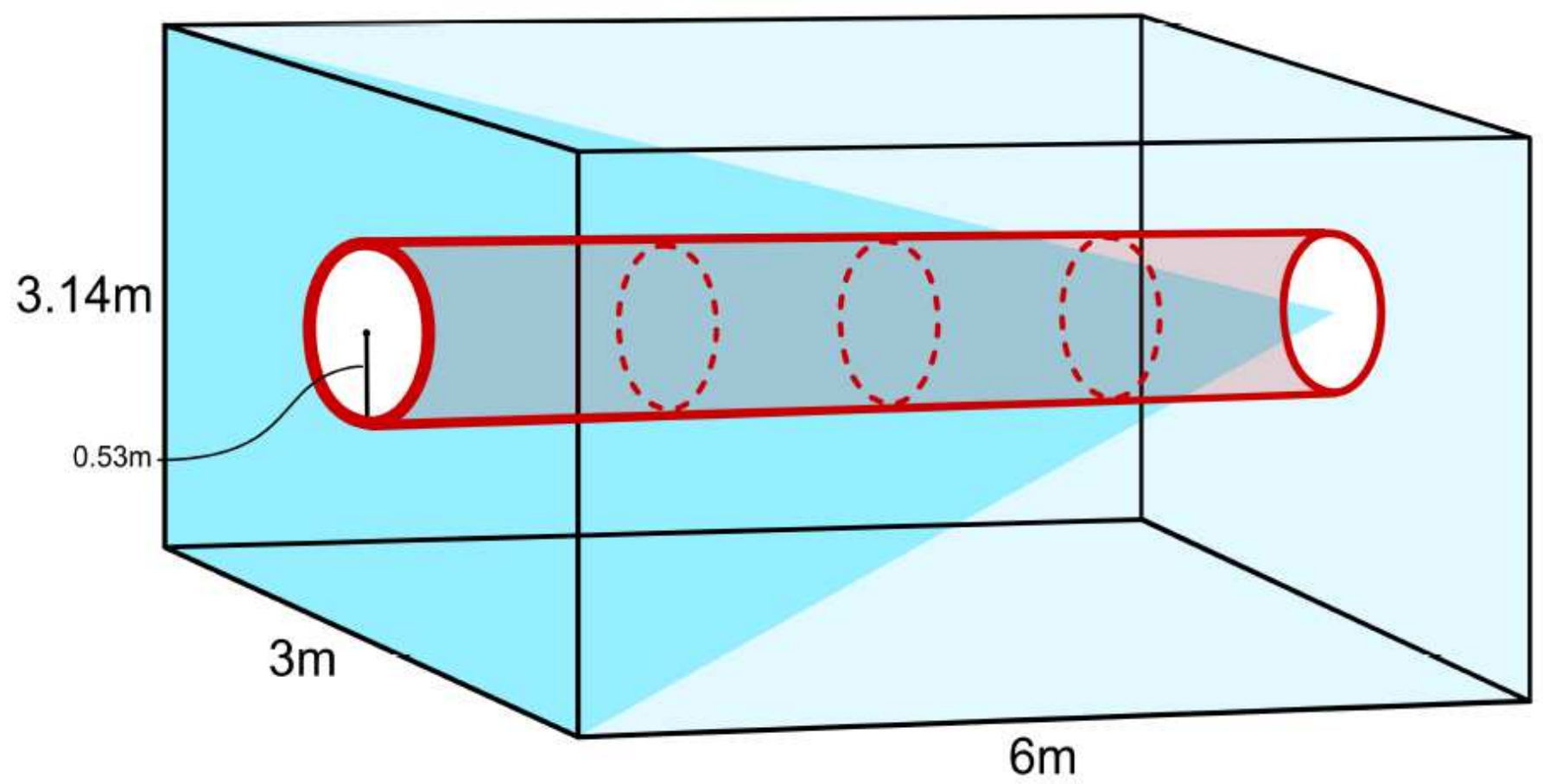

Figure 5

A cartoon (not to scale) of the volume (red cylinder) where the droplet/aerosol clouds propagate and the volume (blue pyramid) that the droplet/aerosol clouds get spread to by the air-conditioning.
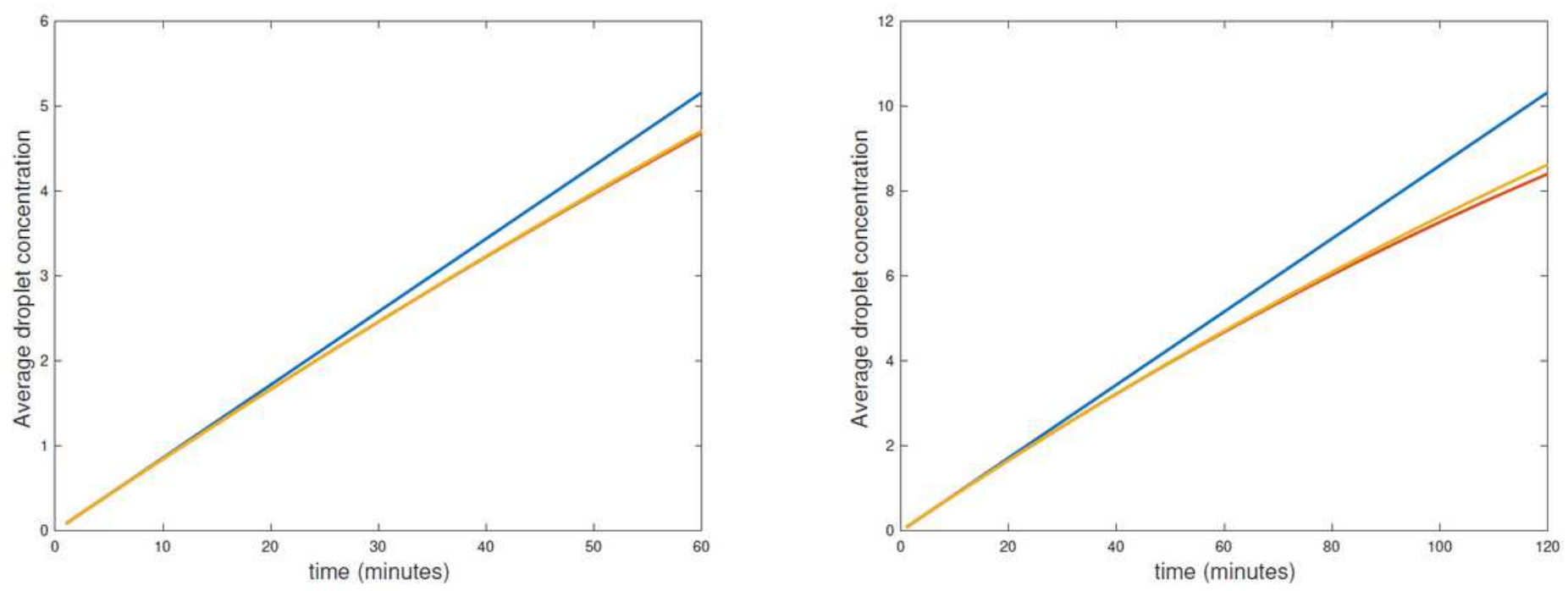

Figure 6

(Left) The aerosol concentration for one hour, top (blue) linear curve, middle (orange) solution of the ODE, bottom (red) the quadratic polynomial. The last two lines are hardly dis-tinguishable. (Right) The aerosol 
concentration for two hours, top (blue) linear curve, middle (orange) solution of the ODE, bottom (red) the quadratic polynomial. The last two lines begin to separate on the right.

\section{Supplementary Files}

This is a list of supplementary files associated with this preprint. Click to download.

- Supplementarylnformation.pdf 